\title{
Escala de soporte social de Tagaya, Okuno, Tamura y Davis: propiedades psicométricas en adultos mayores*
}

\section{The Social Support Scale of Tagaya, Okuno, Tamura and Davis: Psychometric Properties in the Elderly}

\author{
Armando Rivera-Ledesma" \\ Universidad de Londres, México \\ María Montero-López Lena \\ Universidad Nacional Autónoma de \\ México \\ Claudia Iveth Astudillo-García \\ Universidad de Londres, México. \\ Recibido: 5 de diciembre de 2012 \\ Revisado: 4 de abril de 2013 \\ Aceptado: 3 de junio de 2013
}

\section{Resumen}

La presente investigación propone la adaptación al español y validación de la Escala de Soporte Social (ESS-AM) de Tagaya, Okuno, Tamura y Davis (2000), conducida en cuatro muestras independientes de adultos mayores de la Ciudad de México. La escala, de ocho reactivos, mide la percepción de apoyo social mediante un solo factor que explica el $60,7 \%$ de la varianza, con una consistencia interna a de 0,84 y correlaciones negativas significativas con la variable soledad. Con base en ello, se concluye que la ESS-AM es un instrumento confiable, útil y sencillo en funciones de tamizaje para evaluar si el adulto mayor cuenta con adecuados niveles de apoyo social percibido.

Palabras clave: apoyo social, vejez, evaluación, soledad, escala.

\section{Abstract}

This research presents the Spanish adaptation and validation of the Social Support Scale (ESS-AM) from Tagaya, Okuno, Tamura and Davis (2000), conducted in four independent samples of elderly people from Mexico City. The scale, with eight reactive measures about perceived social support, with a single factor

\footnotetext{
Artículo de investigación. Investigación financiada por el Consejo Nacional de Ciencia y Tecnología (México), bajo el No. 95177 y la Universidad de Londres.

** Correspondencia: Armando Rivera-Ledesma, Coordinación de Investigación y Producción Científica. Dirección postal: Tabasco 329, Col. Roma, Delegación Cuauhtémoc, 06700, México, D. F. Tel. 5207-7405. Correo electrónico: armandoriveral@hotmail.com
} 
that explained the $60.7 \%$ of the variance with an internal consistency alpha of .84 and significant negative correlations with the Loneliness. ESS-AM is a reliable, useful and simple, which can be used to assess if elderly people have adequate levels of perceived social support in terms of screening

Keywords: social support, age, evaluation, loneliness, scale.

\section{Introducción}

Los cambios en los patrones de mortalidad y fecundidad de las últimas décadas han provocado importantes alteraciones en el volumen, la dinámica geográfica y la estructura poblacional por edad; parece ser que estamos atravesando un proceso gradual de "envejecimiento demográfico” (Partida, 2005). Esta transición demográfica, de dimensión mundial, tiene por consecuencia no solo una población más numerosa, sino que también vive un mayor número de años (Tuirán, 2002).

La proporción de personas mayores de 60 años está aumentando más rápidamente que cualquier otro grupo de edad. De acuerdo con el informe sobre salud de la Organización Mundial de la Salud (OMS, 2003), en el mundo hay unos 600 millones de personas mayores de 60 años y se calcula que ese número se habrá duplicado en 2025, y en 2050 se habrá transformado en 2000 millones, la mayoría de las cuales se encontrarán en países en desarrollo. En México se estima que, en términos absolutos, por cada 100 niños habrá 166,5 adultos mayores en el 2050 (CONAPO citado en GallegosCarrillo et al., 2009).

Este proceso de envejecimiento, visto como resultado de la interacción de factores biológicos, psicológicos y sociales, se complica con la aparición de patologías que contribuyen en gran medida a la pérdida de capacidades, lo que genera un incremento de las necesidades de salud, que se asocia a su vez con una mayor demanda y utilización de servicios de consulta externa, hospitalización, rehabilitación y apoyo social (INEGI, 2007). Ello significa que la sociedad debe adaptarse a las demandas económicas y sociales necesarias para mejorar al máximo la salud y la capacidad funcional de las personas mayores, su participa- ción social y su seguridad, para lo cual resultará fundamental identificar las condiciones o las variables que faciliten este proceso.

En esta línea, se considera a las redes sociales como un elemento fundamental para el desarrollo integral de las personas, ya que es dentro de estas donde toman sentido los roles sociales, se promueve una sensación de pertenencia y se genera la integración social (Guzmán, Huenchuan y Montes de oca, 2002 en Mendoza-Núñez y Martínez Maldonado, 2009). Por el contrario, contar con redes de apoyo insuficientes se ha relacionado con discapacidad, fragilidad y mortalidad en adultos mayores (Andrew, Mitnitski y Rockwood, 2008; Holt-Lunstad, Smith y Layton, 2010). Los mecanismos que han sido considerados para explicar estos hechos incluyen el papel de los factores fisiológicos, como el estrés, la función inmune y los procesos inflamatorios, que puedan ser exacerbados en aquellos individuos a partir de estresores asociados con bajos niveles de apoyo social o sentimientos de soledad (Andrew et al., 2008).

El apoyo social puede ser entendido como cualquier tipo de soporte sistemático ofrecido por grupos o personas con efectos positivos para el receptor (Da Silva, Melo y Castanheira, 2008). Es un constructo multidimensional formado por dos ámbitos básicos: el área estructural (existencia de redes o vínculos sociales) y el área funcional o expresiva (tipo de apoyo recibido), que puede ser de tres tipos: recursos emocionales, instrumentales e informativos (Dean, 1986, citado en Calvo y Díaz, 2004).

Tardy (1985) describe el apoyo social en función de cinco dimensiones: 1 . dirección, hace referencia a la bidireccionalidad del apoyo social: provisto o recibido; 2 . disposición: hace la distinción entre el apoyo disponible, ante situaciones hipo- 
téticas específicas y el apoyo real que se recibe; 3. descripción/evaluación: el apoyo descrito especifica las acciones o categorías de apoyo; el apoyo evaluado refleja la adecuación de este en términos de satisfacción; 4. contenido: se distinguen tres categorías: emocional, instrumental e informativo, y 5. redes, alude a las características transaccional e interpersonal del apoyo en términos de proveedores, fuentes y relaciones íntimas.

En términos generales, se ha documentado el efecto positivo del apoyo social sobre la mortalidad (Holt-Lunstad et al., 2010), la calidad de vida (Gallegos-Carrillo et al., 2009), la reactividad cardiovascular (Phillips, Gallagher y Carroll, 2009) y específicamente en adultos mayores en la salud funcional (Lachman y Agrigoroaei, 2010), el proceso de la declinación motora (Buchman et al., 2009), el estado cognitivo (Green, Rebok y Lyketsos, 2008), el bienestar (Westaway, Seager, Rheeder y Van, 2005), la disminución de síntomas de depresión (Friedman et al., 2005) y para amortiguar la experiencia solitaria (Montero-López Lena y Rivera-Ledesma, 2009). Respecto al proceso de enfermedad, se ha relacionado con el tiempo del diagnóstico y de adaptación al proceso de enfermedad (Novotny et al., 2010), con el autocuidado (Amador-Díaz, Márquez-Celedonio y Sabido-Sighler, 2007), la adherencia al tratamiento (Stadel, 2003) y una menor intensidad de síntomas (Novotny et al., 2010).

Sin embargo, durante el ciclo vital, el apoyo social experimenta transformaciones no solo cualitativas, sino cuantitativas; la red social en edades avanzadas muestra una serie de cambios asociados con eventos vitales, como la finalización de la crianza de los hijos, el abandono de las relaciones procedentes del ámbito laboral y la disminución del grupo de parientes e iguales por efecto de la mortalidad (Meléndez-Moral, Tomás-Miguel y Navarro-Pardo, 2007), lo que hace que la socialización sea complicada, la pérdida del esposo o de amigos cercanos hace pensar en la hipótesis sobre la vulnerabilidad a perder apoyo social (Gurung, Taylor y Seeman, 2003).

Por estas razones, es importante considerar que además de los cambios fisiológicos, los adultos mayores experimentan una serie de cambios sociales; así, al ser el apoyo social percibido como una variable que se asocia con un mejor estado de salud y de calidad de vida, se vislumbra la importancia de contar con instrumentos validados en contextos latinoamericanos que permitan la medición efectiva del soporte social, tomando en cuenta los cambios cognitivos que también presentan.

En este sentido, se ha señalado que un problema central en la evaluación del adulto mayor es practicar el diagnóstico diferencial entre el envejecimiento normal y el envejecimiento patológico (Ferrante, 2004). Contar con instrumentos fáciles de aplicar e interpretar que se adecuen a las características de este grupo (características cognitivas, demográficas, de escolaridad, de acceso a recursos, etc.), y que ayuden a dilucidar si el adulto mayor cuenta con niveles adecuados de apoyo que le permitan afrontar positivamente los cambios que está teniendo en su vida (pérdidas, condición de enfermedad, etc.), es, pues, una tarea importante.

Tagaya et al., (2000) desarrollaron la Escala de Soporte Social (ESS-AM) ${ }^{1}$ para ser usada específicamente en adultos mayores; esta escala breve, con una consistencia interna a de 0,70, está compuesta por ocho ítems acerca de la disponibilidad percibida de varios grados de apoyo, que incluyeron: soporte emocional, cuidado de corto y largo plazo, soporte económico e instrumental. La escala se desarrolló en un proceso que incluyó una muestra de 1956 adultos mayores japoneses, compuesta con el $60,2 \%$ de mujeres y una media de edad de 78,2 y 85 años para mujeres y hombres, respectivamente. El 93,4\% vivían con sus familias. El presente trabajo tiene por objetivo analizar las propiedades psicométricas de la ESSAM en cuatro muestras independientes de adultos mayores mexicanos provenientes de diferentes contextos. Por su brevedad, la ESS-AM puede resultar beneficiosa en la investigación con adultos mayores en un contexto latinoamericano.

1 'ESS-AM' no son siglas utilizadas por Tagaya et al.; se introducen aquí para identificarla con facilidad y destacar su adaptación en adultos mayores. 


\section{Método}

\section{Muestra}

En total, se incluyeron 502 participantes divididos en cuatro muestras independientes; en cada una se recurrió a un muestreo no probabilístico y se seleccionó a los participantes por su disponibilidad, de acuerdo con los siguientes criterios de inclusión: 1. alfabetas; 2 . edad mínima de 50 años; 3 . funcionales física y cognitivamente en medida suficiente como para contestar por sí mismos el cuestionario; 4. sin problemas de ceguera, sordera o mudez significativas. Las muestras fueron las siguientes:

- Muestra $1\left(M_{1}\right)$ : estuvo compuesta por 193 adultos mayores de la comunidad (COM); 134 mujeres $(69,4 \%)$ y 59 hombres $(30,6 \%)$, con una media de edad de 60,1 (DE = 7,6) y 60,0 años $(\mathrm{DE}=7,9)$, respectivamente, de los cuales 188 $(\mathbf{9 7 , 9 \% )}$ eran cristianos evangélicos. La muestra fue elegida en una congregación cristiana en un periodo que comprendió cuatro domingos.

- Muestra $2\left(M_{2}\right)$ : participaron 104 pacientes, 72 mujeres $(69,2 \%)$ y 32 hombres $(30,8 \%)$, con una media de edad de 64,5 (DE $=2,8)$ y 65,6 años $(\mathrm{DE}=3,1)$, respectivamente, con diagnóstico de diabetes mellitus tipo 2 (DM-2), en tratamiento médico de por lo menos tres meses. Los pacientes fueron captados en la consulta externa del servicio de medicina interna de un hospital de tercer nivel de la Ciudad de México.

- Muestra $3\left(M_{3}\right)$ : un total de 83 pacientes diabéticos con insuficiencia renal crónica (IRC) bajo tratamiento con diálisis peritoneal continua ambulatoria (DPCA); 43 mujeres $(51,8 \%$ ) y 40 hombres $(48,2 \%)$, con una media de edad de 52,2 $(D E=17,1)$ y 54,8 años $(D E=13,3)$, respectivamente; el $40 \%$ de la muestra $(N=33)$ con edades comprendidas entre los 60 y los 82 años. Fueron captados en la unidad de diálisis de un hospital gubernamental de la Ciudad de México mientras acudían a una consulta de seguimiento.

- Muestra $4\left(\mathrm{M}_{4}\right)$ : compuesta por 122 pacientes, 68 mujeres $(55,7 \%)$ y 54 hombres $(44,3 \%)$, con una media de edad de 60,0 (DE = 8,5) y 62 años $(D E=7,9)$, respectivamente, captados en la sala de espera de consulta externa (CE) de una clínica de salud gubernamental. Fueron encuestados mientras aguardaban ser atendidos en una consulta de rutina con fines de seguimiento médico o control farmacológico.

\section{Instrumentos}

El primer instrumento es la Escala de Soporte Social (ESS-AM) (Tagaya et al., 2000). La escala original fue estructurada con ocho ítems, con opciones de respuesta tipo Likert de tres puntos: sí, no e innecesario, y puntajes de 1,0 y 0,5 , respectivamente. Con el fin de facilitar la elección de una opción de respuesta que permitiese identificar una tendencia mejor definida y la graduación de la percepción de apoyo social para su empleo en Latinoamérica, se amplió la escala Likert a cuatro opciones de respuesta, donde a mayor puntaje mayor soporte social percibido. Adicionalmente, esta medida podría tener un efecto positivo en la consistencia interna del instrumento, como ha podido ser evidenciado en otros casos (e. g. McCordie, 1979; Hoogstraten, Koele y Van der Laan, 1998; Rivera-Ledesma y Montero-López Lena, 2009).

El segundo es la Escala de Soledad en el Adulto Mayor (IMSOL-AM, Montero-López Lena y RiveraLedesma, 2009). Originalmente diseñada en México para su aplicación en población general, y posteriormente adaptada para su uso en adultos mayores, consta de 20 reactivos con opciones de respuesta tipo Likert de cinco puntos, donde a mayor puntaje mayor soledad. Contiene dos subescalas que evalúan alienación familiar (entendida como carencia de afecto proveniente de la familia nuclear) e insatisfacción personal (carencia de propósitos en la vida). Se documenta una consistencia interna de 0,89 .

Adicionalmente, se incluyó un cuestionario con preguntas de orden sociodemográfico, cuyo contenido puede consultarse en la tabla 1 .

\section{Procedimiento}

En principio, la ESS-AM fue traducida del inglés al español mexicano siguiendo un procedimiento doble ciego. Cada reactivo fue traducido cuidan- 
do el uso de un lenguaje coloquial y evitando el empleo de enunciados negativos o con doble negación. Ulteriormente, estos enunciados fueron traducidos nuevamente al inglés, y finalmente esta traducción se comparó con los enunciados originales en inglés. Se estimó el porcentaje de acuerdo entre ambas aproximaciones, para lo cual se obtuvo un $100 \%$ de coincidencias.

Las instrucciones fueron: "Lea con atención las siguientes preguntas y elija la respuesta que mejor describa su situación personal". Hecho lo anterior, los instrumentos se aplicaron de forma independiente y secuencial en las cuatro muestras de adultos mayores, previo consentimiento informado para participar en la investigación. Un investigador estuvo a cargo de la aplicación, quien aseguró a los participantes la confidencialidad de los datos. La información recabada fue capturada y analizada en el programa SPSS v. 17.0. Además de análisis descriptivos básicos, se evaluó el instrumento mediante análisis factorial, índice a de Cronbach de consistencia interna y correlación $r$ de Pearson. Para la comparación de los datos sociodemográficos y medias entre muestras se emplearon el análisis de varianza y la prueba $\chi^{2}$.

\section{Resultados}

Las muestras quedaron conformadas con las características sociodemográficas descritas en la tabla 1. Con el fin de determinar la heterogeneidad sociodemográfica de las muestras se compararon sus características; así, se concluyó que estas son diferentes entre sí.

Tabla 1.

Características sociodemográficas de las muestras

\begin{tabular}{|c|c|c|c|c|c|c|c|}
\hline Característica & & $M_{1}(\mathrm{COM})$ & $M_{2}(D M-2)$ & $M_{3}($ IRC) & $M_{4}(C E)$ & Total & Estadístico \\
\hline \multirow{2}{*}{ Sexo } & Hombre & 59 & 32 & 40 & 54 & 185 & \multirow{2}{*}{$\chi^{2}=12,37^{* *}$} \\
\hline & Mujer & 134 & 72 & 43 & 68 & 317 & \\
\hline \multirow{2}{*}{ Edad } & (M) & 60,03 & 55,13 & 53,51 & 60,90 & 58,16 & \multirow{2}{*}{$\mathrm{F}=14,03^{* *}$} \\
\hline & $(D E)$ & $(7,64)$ & $(11,03)$ & $(15,36)$ & $(8,25)$ & $(10,54)$ & \\
\hline \multirow{6}{*}{ Estado Civil } & Soltero & 11 & 40 & 6 & 7 & 64 & \multirow{6}{*}{$\chi^{2}=111,22^{* *}$} \\
\hline & Casado & 90 & 64 & 58 & 86 & 298 & \\
\hline & Unión libre & 4 & 0 & 8 & 4 & 16 & \\
\hline & Separado & 26 & 0 & 2 & 6 & 34 & \\
\hline & Divorciado & 25 & 0 & 1 & 7 & 33 & \\
\hline & Viudo & 37 & 0 & 8 & 12 & 57 & \\
\hline \multirow{6}{*}{ Escolaridad } & Analfabeta & 0 & 12 & 15 & 17 & 44 & \multirow{6}{*}{$\chi^{2}=126,61^{\text {** }}$} \\
\hline & Primaria & 20 & 46 & 23 & 37 & 126 & \\
\hline & Secundaria & 50 & 32 & 22 & 38 & 142 & \\
\hline & Preparatoria & 53 & 12 & 14 & 12 & 91 & \\
\hline & Licenciatura & 22 & 2 & 9 & 14 & 47 & \\
\hline & Posgrado & 48 & 0 & 0 & 4 & 52 & \\
\hline \multirow{4}{*}{ Religión } & Cristiano & 189 & 7 & 2 & 5 & 196 & \multirow{4}{*}{$\chi^{2}=341,87^{* *}$} \\
\hline & Católico & 4 & 94 & 75 & 106 & 185 & \\
\hline & Ninguna & 0 & 3 & 2 & 2 & 4 & \\
\hline & Otra & 0 & 0 & 4 & 9 & 13 & \\
\hline \multirow{2}{*}{ Trabaja } & Sí & 88 & 41 & 16 & 51 & 196 & \multirow{2}{*}{$\chi^{2}=17,01^{* *}$} \\
\hline & No & 105 & 63 & 67 & 71 & 306 & \\
\hline
\end{tabular}

${ }^{* *} \mathrm{p}<0,01 ; M_{1}(\mathrm{COM})$ : muestra comunidad; $M_{2}$ (DM-2): muestra diabéticos tipo $2 ; M_{3}(\mathrm{IRC})$ : muestra insuficiencia renal crónica; $M_{4}(C E)$ : muestra consulta externa. 
Para evaluar el comportamiento estructural de la escala en cada una de las cuatro muestras se llevaron a cabo sendos análisis factoriales por el método de componentes principales con rotación Varimax y autovalores mayores a 1, previa evaluación de adecuación muestral de los datos mediante el índice de Kaiser-Meyer-Olkin (KMO) y la prueba de esfericidad de Bartlett. De forma general, se observaron prácticamente propiedades psicométricas similares en todas las muestras, con excepción de la $M_{3}$ (IRC), donde se obtuvieron valores ligeramente menores, como puede observarse en la tabla 2. Para todos los casos el análisis factorial arrojó un solo factor.

Tabla 2.

Propiedades psicométricas por tipo de muestra

\begin{tabular}{lccccc}
\hline & $\begin{array}{c}\mathrm{M}_{1} \\
(\mathrm{COM})\end{array}$ & $\begin{array}{c}\mathrm{M}_{2} \\
(\mathrm{DM}-2)\end{array}$ & $\begin{array}{c}\mathrm{M}_{3} \\
(\mathrm{IRC})\end{array}$ & $\begin{array}{c}\mathrm{M}_{4} \\
(\mathrm{CE})\end{array}$ & Total \\
\hline KMO & 0,90 & 0,93 & 0,88 & 0,89 & 0,92 \\
$\begin{array}{l}\text { Varianza } \\
\text { explicada }\end{array}$ & 62,9 & 72,8 & 58,5 & 60,8 & 63,6 \\
$\begin{array}{l}\text { a de } \\
\text { Cronbach }\end{array}$ & 0,91 & 0,95 & 0,88 & 0,90 & 0,91 \\
\hline
\end{tabular}

Con el fin de evaluar la estructura de la escala con base en las cuatro muestras $(N=502)$ se llevó a cabo otro análisis factorial con la misma metodología. Este análisis mostró un índice KMO de 0,92 y la prueba de esfericidad de Bartlett fue significativa; el análisis de correlaciones entre ítems arrojó asociaciones que se mantuvieron en un rango de $r=0,40$ a $r=0,56$; todas significativas con $p<0,01$. Con estos resultados previos se concluyó la pertinencia del análisis factorial. Así, la estructura de la escala mostró un único factor, en acuerdo con lo informado en la versión original por Tagaya et al. (2000), que explica un $60,7 \%$ de la varianza con una consistencia interna a de 0,84 . Estos valores fueron superiores a los informados por los autores del instrumento, que en sujetos japoneses ascendieron a 33,8\% de varianza explicada y un a de 0,77 . En la tabla 3 se muestra la matriz de componentes y la saturación de cada ítem en la muestra de 502 adultos mayores mexicanos.
Tabla 3.

ESS-AM carga factorial por reactivo

\begin{tabular}{|c|c|c|}
\hline & Pregunta & $\begin{array}{c}\text { Carga } \\
\text { factorial }\end{array}$ \\
\hline 1 & $\begin{array}{l}\text { ¿Cuenta con alguien a quién } \\
\text { pedirle consejo acerca de sus } \\
\text { preocupaciones? }\end{array}$ & 0,857 \\
\hline 2 & $\begin{array}{l}\text { ¿Cuenta con alguien que le cui- } \\
\text { da cuando usted se enferma? }\end{array}$ & 0,856 \\
\hline 3 & $\begin{array}{l}\text { ¿Cuenta con alguien que se } \\
\text { preocupa por usted? }\end{array}$ & 0,851 \\
\hline 4 & $\begin{array}{l}\text { ¿Cuenta con alguien que le } \\
\text { anima o alienta cuando se } \\
\text { siente mal? }\end{array}$ & 0,847 \\
\hline 5 & $\begin{array}{l}\text { ¿Cuenta con alguien a quién } \\
\text { pedirle dinero cuando lo } \\
\text { necesita? }\end{array}$ & 0,847 \\
\hline 6 & $\begin{array}{l}\text { ¿Cuenta con alguien que le } \\
\text { haga sentir tranquilo? }\end{array}$ & 0,834 \\
\hline 7 & $\begin{array}{l}\text { ¿Cuenta con alguien a quien } \\
\text { pueda pedirle que haga algo } \\
\text { para usted? }\end{array}$ & 0,829 \\
\hline 8 & $\begin{array}{l}\text { ¿Cuenta con alguien que lo } \\
\text { cuidaría si cayera enfermo por } \\
\text { muchos días? }\end{array}$ & 0,664 \\
\hline
\end{tabular}

Evaluada la estructura factorial, se exploró el grado de homogeneidad de las muestras respecto al comportamiento de la ESS-AM. En la figura 1 se observan las características de cada una de ellas y de la muestra total. De forma general, se observó un comportamiento similar entre la $M_{1}(C O M)$, $M_{2}(D M-2)$ y $M_{4}(C E)$; la $M_{3}$ (IRC) fue la que presentó ligeras diferencias en el límite inferior de la caja, así como una menor dispersión de los datos. Se buscaron diferencias estadísticamente significativas entre las medias de las muestras, mediante un análisis de varianza de una sola vía; los resultados permitieron concluir la igualdad entre las medias $(F=1,48, g l=3, p=0,22)$, asumiéndose que la ESS-AM se comportó similarmente en las cuatro muestras, tanto en poblaciones clínicas (de diabéticos, con IRC y de consulta externa; $M_{2}$, $M_{3}, M_{4}$ ) y la no clínica (adultos mayores de la comunidad $M_{1}$ ). 
Figura 1. Puntuaciones de la ESS-AM por tipo de muestra y el total

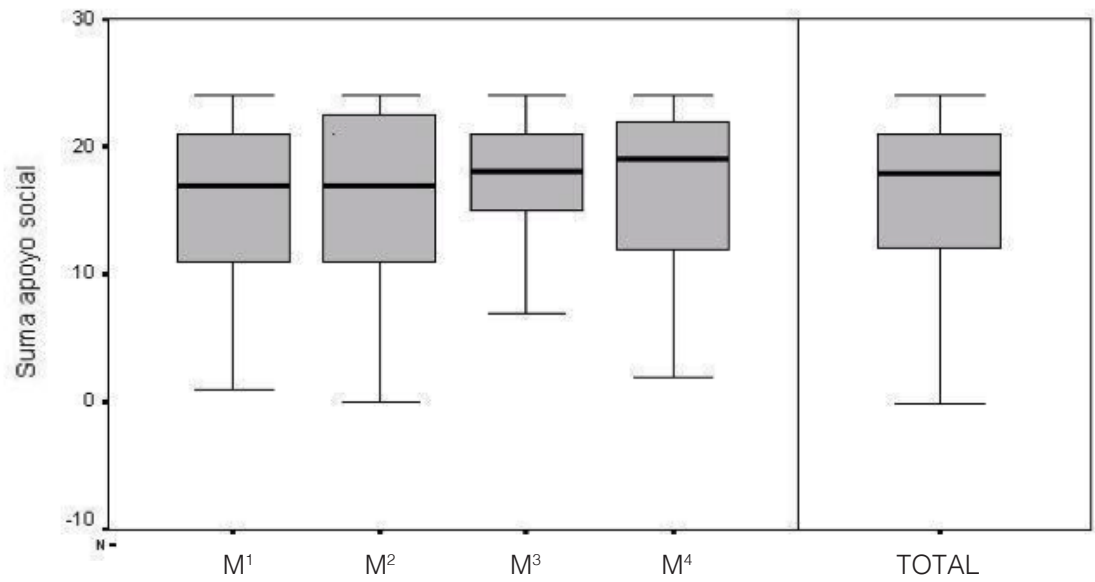

Esta homogeneidad entre las muestras aportó evidencia adicional acerca de la pertinencia de estructurar una sola muestra total con la suma de todas ellas ( $N=502$ adultos mayores), como se hizo inicialmente. Así, se estimaron puntos de corte con base en valores $Z$ estandarizados. En la tabla 4 se muestran las puntuaciones crudas de la ESS-AM, seleccionadas en atención a las principales desviaciones estándar de la distribución.

Tabla 4.

Puntos de corte de la Escala de Soporte Social

\begin{tabular}{clc}
\hline DE & & Puntuación cruda \\
\hline 1 & Superior a la media & $20-24$ \\
0,5 & Normal alto & $17-19$ \\
Media & Normal & 16 \\
$-0,5$ & Normal bajo & $13-15$ \\
-1 & Inferior a la media & $10-12$ \\
-2 & Bajo & $4-9$ \\
-3 & Muy bajo & $0-3$ \\
\hline
\end{tabular}

Por otro lado, con el fin de aportar evidencia sobre la validez de la ESS-AM, se evaluó su asociación con el IMSOL-AM, mediante la correlación producto-momento de Pearson; en todas las muestras se obtuvo una asociación negativa significativa, tan- to para el puntaje total de la escala de soledad, como para sus dos subescalas (tabla 5).

Tabla 5.

Correlaciones entre la ESS-AM y el IMSOL-AM por tipo de muestra

\begin{tabular}{lccccc}
\hline & $\begin{array}{c}M_{1} \\
(C O M)\end{array}$ & $\begin{array}{c}M_{2} \\
(D M-2)\end{array}$ & $\begin{array}{c}M_{3} \\
(I R C)\end{array}$ & $\begin{array}{c}M_{4} \\
(C E)\end{array}$ & Total \\
\hline $\begin{array}{l}\text { IMSOL-AM } \\
\text { total }\end{array}$ & $-0,57^{* *}$ & $-0,50^{* *}$ & $-0,47^{* *}$ & $-0,51^{* *}$ & $-0,51^{* *}$ \\
$\begin{array}{l}\text { Subescalas } \\
\text { IMSOL-AM: }\end{array}$ & & & & & \\
$\begin{array}{l}\text { alienación } \\
\text { familiar }\end{array}$ & $-0,60^{* *}$ & $-0,48^{* *}$ & $-0,50^{* *}$ & $\mathrm{n} / \mathrm{s}$ & $-0,54^{* *}$ \\
$\begin{array}{l}\text { Insatis- } \\
\text { facción } \\
\text { personal }\end{array}$ & $-0,47^{* *}$ & $-0,49^{* *}$ & $-0,40^{* *}$ & $\mathrm{n} / \mathrm{s}$ & $-0,43^{* *}$ \\
\hline
\end{tabular}

${ }^{* *} p<0,01 ; n / s=$ no significativa.

Finalmente, se hicieron comparaciones por el grado de soporte social calculado con base en algunas variables sociodemográficas; no se encontraron diferencias significativas en cuanto a sexo $(t=-0,33$, $g l=412,1, p=0,74)$, escolaridad $(F=0,39, g l=$ $5, p=0,89)$, religión $(\mathrm{F}=0,87, g l=8, p=0,54) \mathrm{y}$ ocupación ( $t=-1,1, g l=424,8, p=0,26)$. El estado civil sí informó diferencias significativas $(F=6,0$, $g l=5, p=0,000$ ), específicamente en las variables soltero $\neq$ divorciado, casado $\neq$ separado, casado $\neq$ divorciado, divorciado $\neq$ viudo (todas con $p<0,05$ ). 


\section{Discusión}

El presente estudio tuvo como objetivo evaluar las propiedades psicométricas de la Escala de Soporte Social de Tagaya et al. (2000) en cuatro muestras de adultos mayores mexicanos. De acuerdo con los resultados obtenidos, la escala presenta adecuadas propiedades psicométricas, incluso superiores a lo informado por los autores del instrumento original. Estos hallazgos sugieren que la ESS-AM es un instrumento válido, breve y de fácil aplicación en adultos mayores. Esto es de particular importancia en la comunidad psicogerontológica, pues se ha exhortado a desarrollar instrumentos amables en su aplicación y con buenas propiedades psicométricas (Meléndez-Moral et al., 2007).

Al aplicar la ESS-AM a cuatro muestras independientes y diferentes sociodemográficamente, compararlas con base en su contexto clínico (enfermos crónicos frente a adultos mayores de la comunidad) y conservar sus propiedades psicométricas se da cuenta de su validez externa. A su vez, encontrar diferencias significativas en la cantidad de soporte recibido entre solteros y casados (tal como lo informado por Meléndez-Moral et al., 2007), así como el asociarse negativamente con la medición de soledad, aportan criterios de validez adicional, dado que se ha documentado que individuos con contactos sociales limitados están en riesgo de sufrir aislamiento social (Findlay, 2003).

La estructura de la ESS-AM explora, de una forma sencilla y breve, y en un solo factor compuesto por los ocho reactivos del instrumento, las dimensiones de disposición (apoyo percibido) y descripción/evaluación (acciones específicas de apoyo y frecuencia), propuestas por Tardy (1985). La ESSAM parece centrarse con mayor especificidad en las categorías de contenido (apoyo emocional: reactivos 3,4 y 6; instrumental: reactivos 2, 5, 7 y 8; e informativo: reactivo 1), propuestas por este autor. La escala resulta útil como medida de tamizaje para determinar si el adulto mayor posee una percepción adecuada de soporte social, pues ha sido ampliamente documentado que percibir una red social fuerte influirá directamente sobre la calidad de vida (Gallegos-Carrillo et al., 2009), el estado de salud (Lachman y Agrigoroaei, 2010) y la adaptación en el proceso de enfermedad (Novotny et al., 2010).

Un hecho de particular importancia fue no encontrar diferencias por sexo como las informadas por Gurung et al. (2003), Meléndez-Moral et al. (2007) y Rodríguez-Laso, Zunzunegui y Otero (2007), lo que podría indicar un posible efecto homogeneizador de la vejez de estos adultos mayores mexicanos sobre el entorno social de hombres y mujeres, tal como lo plantearon Escobar, Puga y Martín (2008).

No obstante, si bien la relación entre apoyo social y sus efectos benéficos parece ser clara, existen otras variables importantes por identificar que no son exploradas por la escala y que deberían tomarse en cuenta para futuras investigaciones.

Entre las cuestiones importantes por considerar está incluir reactivos que permitan conocer la red específica a la que es atribuido el soporte social percibido (familia, pareja, amigos, etc.), o las diferencias cualitativas entre ellas. Por ejemplo, en una investigación con 439 adultos mayores casados se encontró que los hombres reciben apoyo emocional principalmente de sus esposas, mientras que las mujeres lo hacen mayormente de sus amigos, parientes e hijos (Gurung et al., 2003). Otros aspectos importantes serían aportar datos acerca de la cantidad de apoyo social real para contrastar la información cualitativa a menudo fundamental para fines subjetivos terapéuticos (Green et al., 2008), así como dilucidar los factores que influyen en la percepción de apoyo social y el grado de autoeficacia para solicitarlo a quienes puedan proveerlo (Calvo y Díaz, 2004).

A su vez, es importante tener presente que el diseño del presente estudio, al ser transversal, solo explora un momento en el tiempo en relación con la cantidad de apoyo del adulto mayor, pero es posible deducir que este puede experimentar cambios importantes a lo largo de dicha etapa de la vida. En este sentido, es necesario considerar los cambios surgidos en las relaciones sociales como parte del proceso de transición sociodemográfica, que ha extendido el papel que padres 
e hijos desempeñan en la dinámica familiar; los ancianos viven más y esto impacta sus vidas, haciendo necesaria una redefinición de sus funciones y responsabilidades (cf. Tuirán, 2002); o por el contrario, observar la cantidad, cada vez mayor, de ancianos que viven solos (Robles, 2006).

Estas nuevas relaciones se encuentran en un proceso, aún incipiente, de redefinición, por lo que es necesario considerar estos cambios de las estructuras familiares en las estrategias de intervención; por ejemplo, reforzar el soporte social percibido proveniente tanto de la familia, como de redes sociales alternativas (amigos, compañeros, organizaciones civiles, etc.), en atención a la sugerencia de que son estos últimos los que pueden influir positivamente sobre el estado de salud del sujeto y que, al parecer, son más fáciles de modificar que las relaciones de lazos de parentesco (Jorm, 2005).

Sin embargo, en una estructura ideal, un apoyo social óptimo para los adultos mayores estaría compuesto por un sistema abierto, multicéntrico, integrado por componentes informales (familia, amigos, comunidad) y formales (profesionistas e instituciones), matizados por adecuadas conductas de apego (Mendoza-Núñez y Martínez-Maldonado, 2009). En relación con este último concepto, Meléndez-Moral et al. han definido la conducta de apego como "un vínculo afectivo duradero de carácter singular, que se desarrolla, prospera y consolida por medio de la relación activa y recíproca de las partes participantes” (2007, p. 410).

Esta acción recíproca derivada del apego contribuye a la formación de redes sociales entre proveedores y receptores de apoyo, por lo que debe dotarse de un carácter más activo al proceso del envejecimiento para consolidar estas redes de apoyo, más que la sola recepción de estas. En adultos jóvenes (López, Rivera y Reyes, 2007) se ha identificado que es la conducta de apoyo (proveer apoyo social, confort, ayuda para resolver problemas y celebrar éxitos) lo que mantiene la relación de amistad; sin embargo, puede que en adultos mayores esta pueda percibirse de forma diferente. Al respecto, Robles (2006) señala que el hecho de mirar la vejez como un proceso implica notar sus cambios, su ritmo y su dirección para construirla como tal, y que el único medio para llegar a conocer la realidad del adulto mayor contemporáneo es por medio de la indagación empírica, la cual permitirá proveer de alternativas. Por ello, es de suma importancia estudiar este proceso, indagar sobre la realidad del anciano y diseñar instrumentos de evaluación que de una forma sencilla y adecuada a sus características permita conocer su realidad: "el estudio de la vejez, lejos de estar agotado, está abierto a múltiples posibilidades analíticas” (Robles, 2006, p. 165).

En este sentido, la ESS-AM se muestra como una escala útil, breve y sencilla que permitirá evaluar las condiciones en las cuales se encuentra el adulto mayor respecto al nivel de apoyo social que percibe, como una vía para facilitar una mayor capacidad funcional, estado de salud y participación social en este grupo etario.

\section{Referencias}

Amador-Díaz, M. B., Márquez-Celedonio, F. G., \& Sabido-Sighler, A. S. (2007). Factores asociados al auto-cuidado de la salud en pacientes diabéticos tipo 2. Archivos en Medicina Familiar, 9(2), 99-45.

Andrew, M. K., Mitnitski, A. B., \& Rockwood, K. (2008). Social vulnerability, frailty and mortality in elderly people. PLOS ONE, 3, 2232.

Buchman, A. S., Boyle, P. A., Wilson, R. S., Fleischman, D. A., Leurgans, S., \& Bennett, D. A. (2009). Association between late-life social activity and motor decline in older adults. Archives of Internal Medicine, 169(12), 11391146.

Calvo, F., \& Díaz, M. D. (2004). Apoyo social percibido: características psicométricas del cuestionario Caspe en una población urbana geriátrica. Psicothema, 16(4), 570-575.

Da Silva P. I., Melo, R. S., \& Castanheira, N. L. (2008). Red y apoyo social en enfermería familiar: revisión de conceptos. Revista LatinoAmericana Enfermagem, 16(2), 110-117. 
Escobar, M. A., Puga, D., \& Martín, M. (2008). Asociaciones entre la red social y la discapacidad al comienzo de la vejez en las ciudades de Madrid y Barcelona en 2005. Revista Española de Salud Pública, 82(6), 637-651.

Ferrante, V. (2004). Indicadores cognitivos y perceptivo-motores de envejecimiento. Revista Iberoamericana de Diagnóstico y Evaluación Psicológica, 18(2), 137-159.

Findlay, R. A. (2003). Interventions to reduce social isolation amongst older people: where is the evidence? Aging y Society, 23, 647-658. DOI: $10.1017 /$ S0144686X03001296

Friedman, L. C., Brown, A. E, Romero, C., Dulay, M. F, Peterson, L. E, Wehrman, P., Whisnand, D. J., Laufman, L., \& Lomax, J. (2005). Depressed mood and social support as predictors of quality of life in women receiving home health care. Quality of Life Research, 14, 1925-1929.

Gallegos-Carrillo, K., Mudgal, J., Sánchez-García, S., Wagner, F. A., Gallo, J. J., Salmerón, J., \& García-Peña, C. (2009). Social networks and health-related quality of life: a population based study among older adults. Salud Publica de México, 51(1), 6-13.

Green, A. F., Rebok, G., \& Lyketsos, C. G. (2008). Influence of social network characteristics on cognition and functional status with aging. International Journal of Geriatric Psychiatric, 23(9), 972-978. DOI: 10.1002/gps.2023.

Gurung, R. A., Taylor, S. E., \& Seeman, T. E. (2003). Accounting for changes in social support among married older adults: insights from the MacArthur Studies of Successful Aging. Psychology and Aging, 18(3), 487-496. DOI: $10.1037 / 0882-7974.18 .3 .487$.

Holt-Lunstad, J., Smith, T. B., \& Layton, J. B. (2010). Social relationships and mortality risk: a meta-analytic review. PLoS Med, 7(7). DOI:10.1371/journal.pmed.1000316

Hoogstraten, J., Koele, P., \& Van der Laan, J. (1998). Templer's death anxiety scale revisi- ted: the Dutch version. Perceptual and Motor Skills, 87, 1259-1264.

Instituto Nacional de Estadística y Geografía (INEGI). (2007). Recuperado de http://www.inegi.gob.mx/inegi/contenidos/espanol/prensa/ contenidos/estadisticas/2007/edad07.pdf

Jorm, A. F. (2005). Social networks and health: it's time for an intervention trial. Journal of Epidemiology Community Health, 59, 537538.

Lachman, M. E., \& Agrigoroaei, S. (2010). Promoting health in midlife and old age: long term protective effects of control beliefs, social support, and physical exercise. PloS ONE, 10(5).

López, C., Rivera, S., \& Reyes, I. (2007). Inventario de estrategias de mantenimiento de la amistad. Revista Iberoamericana de Diagnóstico y Evaluación Psicológica, 23(1), 23-39.

McCordie, W. R. (1979). Improving measurement of death anxiety. Psychological Reports, 44, 975-980.

Meléndez-Moral, J. C., Tomás-Miguel, J. M., \& Navarro-Pardo, E. (2007). Análisis de las redes sociales en la vejez a través de la entrevista Manheim. Salud Pública de México, 49(6), 408-414.

Mendoza-Núñez, V. M., \& Martínez-Maldonado, M. L. (2009). Escala de Redes de Apoyo Social para Adultos Mayores (ERASAM). En: González-Celis, A.L. (Ed.) Evaluación en Psicogerontología (pp. 95-112). México: El Manual Moderno.

Montero-López Lena, M., \& Rivera-Ledesma, A. (2009). IMSOL-AM: Escala de Soledad en el Adulto Mayor. En: González-Celis, A. L. (Ed.) Evaluación en Psicogerontología (pp. 123131). México: El Manual Moderno.

Novotny, P. J., Smith, D. J., Guse, L., Rummans, T. A., Hartmann, L., Alberts, S., Goldberg, R., Gregory, D., Johnson, M., \& Sloan, J. A. 
(2010). A pilot study assessing social support among cancer patients enrolled on clinical trials: a comparison of younger versus older adults. Cancer Management and Research, 2, 133-142.

Organización Mundial de la Salud. OMS (2003). Informes sobre la salud en el mundo. Recuperado de http://whqlibdoc.who.int/ whr/2003/9243562436.pdf

Partida, V. (2005). La transición demográfica y el proceso de envejecimiento en México. Papeles de Población, 45, 9-27.

Phillips, A. C., Gallagher, S., \& Carroll, D. (2009). Social support, social intimacy, and cardiovascular reactions to acute psychological stress. Annals of Behavioral Medicine, 37, 3845. DOI: $10.1007 / \mathrm{s} 12160-008-9077-0$

Rivera-Ledesma, A., \& Montero-López Lena, M. (2009). Propiedades psicométricas de la escala de ansiedad ante la muerte de Templer en sujetos mexicanos. Diversitas, 6(1), 135140.

Robles, L. (2006). La vejez: nuevos actores, relaciones sociales y demandas políticas. Relaciones, 105(27), 140-175.
Rodriguez-Laso, A., Zunzunegui, M. V., \& Otero, A. (2007). The effect of social relationships on survival in elderly residents of a Southern European community: a cohort study. BMC Geriatrics, 7(19). DOI: 10.1186/1471-2318-7-19.

Stadel, S. (2003). Rural elders with chronic disease: place of residence, social network, social support and medication adherence. New York: Dissertation of the State University.

Tagaya, A., Okuno, S., Tamura, M., \& Davis, A. (2000). Social support and end-of-life issues for small town Japanese elderly. Nursing and Health Sciences, 2, 131-137.

Tardy, C. H. H. (1985). Social suport measurement. American Journal of Community Psycology, 13(2). 187-202.

Tuirán, R. (2002) Transición demográfica, trayectorias de vida y desigualdad social en México: lecciones y opciones. Papeles de Población, 31.

Westaway, M., Seager, J., Rheeder, P., \& Van D. (2005). The effects of social support on health, well-being and management of diabetes mellitus: a black south african perspective. Ethnicity and Health, 10, 73-89. 